\title{
Transmission Latency and Reliability Trade-off in Path-Time Coded Cognitive Radio Ad Hoc Networks
}

\author{
Yi-Chi Chen ${ }^{1}$, I-Wei Lai ${ }^{2}$, Kwang-Cheng Chen ${ }^{1}$, Wen-Tsuen Chen ${ }^{3}$, and Chia-Han Lee ${ }^{2}$ \\ Graduate Institute of Communication Engineering, National Taiwan University, Taiwan ${ }^{1}$ \\ Research Center for Information Technology Innovation, Academia Sinica, Taiwan ${ }^{2}$ \\ Institute of Information Science, Academia Sinica, Taiwan ${ }^{3}$ \\ E-mails: willness1986@gmail.com, iweilai@citi.sinica.edu.tw \\ ckc@ntu.edu.tw, chenwt@iis.sinica.edu.tw, and chiahan@citi.sinica.edu.tw
}

\begin{abstract}
Cognitive radio ad hoc network (CRAHN) is considered as a key technology to enhance the spectrum efficiency for diverse applications. However, due to the opportunistic links, the intrinsic randomness of the CRAHN makes the traditional precise control of the end-to-end transmission unscalable and generally infeasible. The recently-proposed virtual multiple-input multipleoutput (MIMO) framework exploits the multipath routing to create the diversity at the network layer. With only local information and no feedback control channel, the path-time code (PTC) of the virtual MIMO system is able to efficiently provide the error resilient end-to-end transmission. In particular, while the transmission latency should be minimized, more attempts for accessing the opportunistic links can be made if a larger latency is allowed, which improves the error rate performance of the end-to-end transmission. By theoretically analyzing the error rate performance and erasure statistics, we propose a design guideline to determine the waiting period as the limit of the transmission latency. This designed waiting period not only preserves the diversity gained by the PTC but also provides a low transmission latency, resulting in a good balance of the reliability-latency trade-off of the end-to-end PTC transmission in CRAHNs.
\end{abstract}

Index Terms - Cognitive radio ad hoc networks (CRAHNs), virtual multiple-input and multiple-output (MIMO), space-time code (STC), path-time code (PTC), PEP analysis, erasure channel.

\section{INTRODUCTION}

The cognitive radio (CR) enhances the spectrum utilization [1], [2] by permitting the CR users to dynamically access the spectrum without interfering with the primary users. Although the cognitive radio ad hoc network (CRAHN), formed by primary and CR users, greatly enhances the spectrum efficiency, it faces the challenges such as the opportunistic CR links. Specifically, each relay node has to buffer the packet until the link to its neighboring node is available, i.e., no primary users or other CR users currently occupy this link. Therefore, a packet may experience long transmission delay before arriving at the destination node, especially when the spectrum is heavily loaded. To avoid infinite delay and overflow problems, the destination node sets a waiting period as the limit of the end-to-end transmission latency. The packets experiencing a transmission latency beyond the waiting period are discarded before arriving at the destination node [3], leading to the transmission outages. The outage transmission not only deteriorates the error rate performance of the end-toend transmission, but also makes the links unidirectional [4], [5].

Conventionally, many routing algorithms are designed for the precise control of the end-to-end transmission with the assumption of complete end-to-end information [6], [7]. However, since the opportunistic links are unidirectional and unreliable, the exchange of the end-to-end information requires large control overhead [3], [8]. Such huge control overhead makes these algorithms inefficient and unscalable. Recently, the path-time code (PTC) is applied to the multihop multipath cognitive radio network through the virtual multipleinput multiple-output (MIMO) formulation [4], [5]. By using multipath routing and local information [9], [10], the reliable end-to-end PTC transmission is realized efficiently. However, despite the extensive system performance analysis provided in [5], the trade-off among the performance metrics, e.g., the transmission reliability and transmission latency, remains open.

In this work, we focus on the analysis of the waiting period which can be used to trade the transmission reliability with the transmission latency. While a longer waiting period results in lower error rate and longer latency, the shorter waiting period allows shorter transmission latency at the expense of the error rate performance degradation. By deriving the pairwise error probability (PEP), we first analyze the diversity loss caused by the transmission outage. It should be emphasized that the diversity indicates the error rate enhancement with respect to the signal-to-noise ratio (SNR) and thus is an essential metric for the transmission reliability [11]. Then, the relationship between the transmission outage and the waiting period is established and approximated by the Chernoff bound. Based on these two results, the transmission latency, which is proportional to the waiting period, and the reliability, which is reflected by the diversity, are linked, and a design guideline is proposed to strike a good balance between the transmission reliability and latency. Simulation results show that by using our design guideline, the transmission latency can be minimized under the condition of negligible diversity loss.

The paper is organized as follows. We revisit the virtual 
MIMO framework with PTC for CRAHNs in Sec. II. In Sec. III, we derive the relationship between the waiting period and the diversity loss. A design guideline is proposed to minimize the waiting period under the condition of negligible diversity loss. We verify the proposed design guideline by means of Monte Carlo numerical simulations in Sec. IV and draw the conclusions in Sec. V.

\section{SySTEM MODEL}

Let the link denote the connection between two nodes, and path the end-to-end connection route between a pair of source and destination nodes. In this work, we consider multihop networking with $R$ link-disjoint paths [4], [5], [12], [13], each including $N_{r}-1$ relay nodes, $r \in\{1, \ldots, R\}$ as illustrated by an equivalent directed-graph model in Fig. 1. Such multihop/multipath routing can be established using the algorithms proposed in [14], [15].

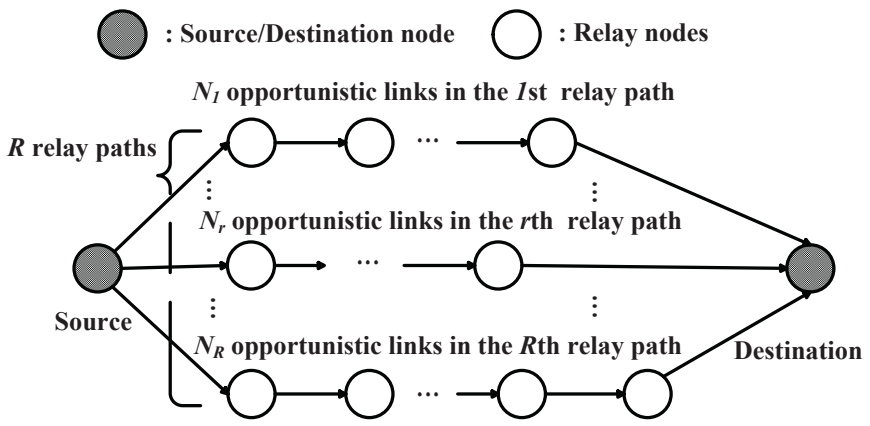

Fig. 1. The equivalent directed graph model of the multihop multipath endto-end transmission in CRAHNs.

At the source node of the CRAHN, $B$ coded packets $\mathbf{s}_{b}=$ $\left[s_{b, 1}, \ldots, s_{b, R}\right]^{\top} \in \mathbb{C}^{R \times 1}$, where $(\cdot)^{\top}$ as the transposition, are transmitted through $R$ relay paths at the $b$ th time instant. These $B$ coded packets are used to encode the same data packet $\mathbf{x}=\left[x_{1}, \ldots, x_{B}\right]^{\top}$ by the coding matrix $\mathbf{C}_{b} \in \mathbb{C}^{R \times B}$ as follows

$$
\mathbf{s}_{b}=\mathbf{C}_{b} \mathbf{x}, \quad b=1, \ldots, B .
$$

Different designs of the coding matrix $\mathbf{C}_{b}$ are addressed in [4], [5] for various channels and performance requirements. In this work, we adopt the discrete Fourier transform (DFT)based PTC, which delivers the best error rate performance of the end-to-end transmission under the fast-fading channels. Letting $\mathbf{C}=\left[\mathbf{C}_{1}^{\top}, \ldots, \mathbf{C}_{B}^{\top}\right]^{\top} \in \mathbb{C}^{R B \times B}$, the $(k, b)$ th entry of $\mathrm{C}$ of the DFT-based PTC is given by

$$
C_{k, b}=\frac{1}{\sqrt{R}} e^{\frac{-j 2 \pi k b}{R B}}, \quad k=1, \ldots, R B, \quad b=1, \ldots, B .
$$

The coded packets then propagate through the $R$ relay paths, i.e., $s_{b, r}$ for the $r$ th relay path at the $b$ th time instant.

We specify the waiting period as the limit of the end-toend transmission latency that the destination node can tolerate. Assuming that the relay nodes periodically sense the availability of the opportunistic link at every predefined sensing interval. If a link is available, the coded packet is forwarded to the next relay node, assuming negligible propagation latency compared with the sensing interval. If the link is currently unavailable, the coded packet is buffered until the beginning of the next sensing interval. We define $T$ as the waiting period which is the time interval normalized with respect to the sensing interval. This implies that each $s_{b, r}$ is allowed to have $T$ attempts to be transported through $N_{r}$ opportunistic links along the $r$ th relay path. Once the end-to-end transmission latency exceeds the waiting period, the coded packet $s_{b, r}$ is discarded, resulting in a transmission outage. We define erasure vector $\mathbf{v}_{b}=\left[v_{b, 1}, \ldots, v_{b, R}\right]^{\top} \in\{0,1\}^{R}$ to describe such transmission outage at the $b$ th time instant, for $b=1, \ldots, B$. This erasure vector comprises $R$ Bernoulli random variables $v_{b, r} \in\{0,1\}$, each indicating whether $s_{b, r}$ successfully arrives at the destination node or not. We define the path fading gain vector $\mathbf{h}_{b}=\left[h_{b, 1}, \ldots, h_{b, R}\right]^{\top} \in \mathbb{C}^{R}$ at the $b$ th time instant, in which each entry is the product of $N_{r}$ link gains. We then can use the Schur product $\mathbf{v}_{b} \circ \mathbf{h}_{b}=\left[v_{b, 1} h_{b, 1}, \ldots, v_{b, R} h_{b, R}\right]^{\top}$ to express the effective path fading gain. With these definitions, the end-to-end PTC transmission at the $b$ th time instant can be formulated as

$$
\begin{aligned}
y_{b} & =\left(\mathbf{v}_{b} \circ \mathbf{h}_{b}\right)^{\top} \mathbf{C}_{b} \mathbf{x}+\sum_{r=1}^{R} \tilde{\eta}_{b, r} \\
& =\mathbf{h}_{b}^{\top} \operatorname{diag}\left(\mathbf{v}_{b}\right) \mathbf{C}_{b} \mathbf{x}+\eta_{b}=\mathbf{h}_{b}^{\top}\left(\mathbf{v}_{b} \circ \mathbf{s}_{b}\right)+\eta_{b},
\end{aligned}
$$

where $y_{b}$ is the received coded packet, $\operatorname{diag}\left(\mathbf{v}_{b}\right)$ is a diagonal matrix whose diagonal entries are $\mathbf{v}_{b}$, and $\tilde{\eta}_{b, r}$ is the additive white Gaussian noise (AWGN) aggregated from all links in the $r$ th path. Due to different AWGN noises $\tilde{\eta}_{b, r}$ for every $b$ and $r$, the noise $\eta_{b}$ has time-varying noise power spectrum density $N_{0, b}$.

Now, by stacking the received coded packets $\mathbf{y}=$ $\left[y_{1}, \ldots, y_{B}\right]^{T} \in \mathbb{C}^{B}$ from $b=1$ to $b=B$, the end-to-end PTC transmission in (4) can be reformulated as

$$
\begin{aligned}
\mathbf{y} & =\underbrace{\left[\begin{array}{ccc}
\mathbf{h}_{1}^{\top} & \ldots & \mathbf{0}^{\top} \\
\vdots & \ddots & \vdots \\
\mathbf{0}^{\top} & \ldots & \mathbf{h}_{B}^{\top}
\end{array}\right]}_{\mathbf{H}}\left[\begin{array}{c}
\left(\mathbf{v}_{1} \circ \mathbf{s}_{1}\right) \\
\vdots \\
\left(\mathbf{v}_{B} \circ \mathbf{s}_{B}\right)
\end{array}\right]+\left[\begin{array}{c}
\eta_{1} \\
\vdots \\
\eta_{B}
\end{array}\right] \\
& =\mathbf{H} \cdot(\mathbf{v} \circ \mathbf{s})+\boldsymbol{\eta},
\end{aligned}
$$

where $\mathbf{H} \in \mathbb{C}^{B \times R B}, \mathbf{v}=\left[\mathbf{v}_{1}^{\top}, \ldots, \mathbf{v}_{B}^{\top}\right]^{\top} \in\{0,1\}^{R B}$, $\mathbf{s}=\left[\mathbf{s}_{1}^{\top}, \ldots, \mathbf{s}_{b}^{\top}\right]^{\top} \in \mathbb{C}^{R B}$, and $\boldsymbol{\eta}=\left[\eta_{1}, \ldots, \eta_{B}\right]^{\top} \in \mathbb{C}^{B}$ are the matrix/vector representation of variables for all time instants. From (6), the end-to-end PTC transmission in the CRAHN is described by the same mathematical expression as a MIMO system, where multiple nodes are coordinated to form multihop/multipath routes between a source and destination node pair, where the source node encodes the data along time and path coordinates to gain the diversity [4], [5].

At the destination node, multiple PTC-coded packets arriving at the destination node from various time instants and relay paths are collected. The maximum a posteriori (MAP) criterion is used to jointly detect the data packet $\mathrm{x}$ and the erasure vector $\mathbf{v}_{b}, b=1, \ldots, B$ [5]. From (6), we can see that once more erasures occur, fewer coded packets can be received by the destination node, thereby degrading the error rate performance. Although enlarging the waiting period can reduce the erasure probability $P\left(v_{b, r}=0\right)$, for $b=1, \ldots, B$ 


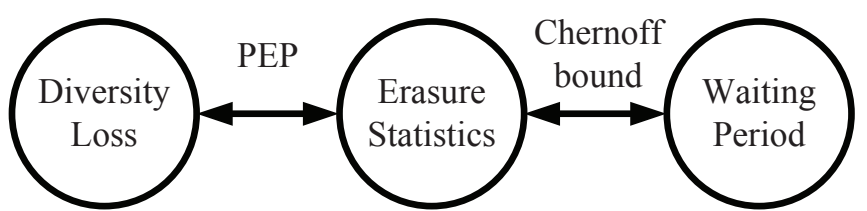

Fig. 2. The relationships among transmission reliability (diversity), erasure statistics, and transmission latency (waiting period).

and $r=1, \ldots, R$ due to allowing more chances of accessing the opportunistic link, the transmission latency is also increased. Consequently, the design of the waiting period is essential to the trade-off between the transmission latency and reliability.

\section{Design Guideline OF THE WAiting PERIOD}

In this section, the PEP is first derived so that we can analyze the diversity loss caused by erasures. Then, the relationship between the erasure statistics and the waiting period is established and approximated by the Chernoff bound. By using these results, the diversity loss and the waiting period which respectively reflect the transmission reliability and the transmission latency, can be connected and utilized to guide the design of the waiting period. The relationships among transmission reliability (diversity), erasure statistics, and transmission latency (waiting period) are illustrated in Fig. 2.

\section{A. Error Performance and Diversity}

Denoting the transmit coded packet and the erroneouslydetected packet with erasure vector by $\mathbf{s}$ and $\hat{\mathbf{s}}$, respectively, the PEP $f(\mathbf{s} \rightarrow \hat{\mathbf{s}})$ is defined as

$$
f(\mathbf{s} \rightarrow \hat{\mathbf{s}})=P(\Lambda(\mathbf{s}, \hat{\mathbf{s}})<0),
$$

where $\Lambda$ denotes the log-likelihood ratio (LLR) of $P(\mathbf{y} \mid \mathbf{s})$ and $P(\mathbf{y} \mid \hat{\mathbf{s}})$ i.e.,

$$
\Lambda=\log P(\mathbf{y} \mid \mathbf{s})-\log P(\mathbf{y} \mid \hat{\mathbf{s}}),
$$

and

$$
P(\mathbf{y} \mid \mathbf{s})=\frac{1}{\pi^{B} \prod_{b=1}^{B} N_{0, b}} e^{-\|\operatorname{diag}(\boldsymbol{\rho})[\mathbf{y}-\mathbf{H} \cdot(\mathbf{v} \circ \mathbf{s})]\|^{2}} ;
$$

$\operatorname{diag}(\boldsymbol{\rho})$ is a diagonal matrix whose diagonal entries are $\boldsymbol{\rho}=$ $\left[1 / \sqrt{N_{0,1}}, \ldots, 1 / \sqrt{N_{0, B}}\right]^{\top}$ [16] . Inserting (9) into (10), we obtain

$\Lambda(\mathbf{s}, \hat{\mathbf{s}})$

$=\|\operatorname{diag}(\boldsymbol{\rho})[\mathbf{y}-\mathbf{H} \cdot(\mathbf{v} \circ \hat{\mathbf{s}})]\|^{2}-\|\operatorname{diag}(\boldsymbol{\rho})[\mathbf{y}-\mathbf{H} \cdot(\mathbf{v} \circ \mathbf{s})]\|^{2}$

$=\|\operatorname{diag}(\boldsymbol{\rho})[\mathbf{H} \cdot(\mathbf{v} \circ(\mathbf{s}-\hat{\mathbf{s}}))]\|^{2}$

$+2 \operatorname{Re}\left\{\operatorname{diag}(\boldsymbol{\rho})\left[\mathbf{H} \cdot\left(\mathbf{v} \circ(\mathbf{s}-\hat{\mathbf{s}})^{H}\right) \boldsymbol{\eta}\right]\right\}$.

where $(\cdot)^{H}$ denotes Hermitian operation, and $\operatorname{Re}\{\cdot\}$ extracts the real part. From (10) we know that given $\mathbf{H}, \Lambda$ is a conditional Gaussian random variable with mean

$$
\mu_{\Lambda}=\|\operatorname{diag}(\boldsymbol{\rho})[\mathbf{H} \cdot(\mathbf{v} \circ(\mathbf{s}-\hat{\mathbf{s}}))]\|^{2}
$$

and variance

$$
\sigma_{\Lambda}^{2}=2 \mu_{\Lambda}
$$

The conditional moment-generating function (MGF) of this Gaussian random variable $\Lambda$ is given by

$$
M_{\Lambda}(t \mid \mathbf{H})=e^{\left(t+t^{2}\right) \mu_{\Lambda}}
$$

Now, to expand (13), we rewrite $\mu_{\Lambda}$ in (11) as

$$
\begin{aligned}
\mu_{\Lambda} & =\sum_{b=1}^{B} \frac{1}{N_{0, b}} \mathbf{h}_{b}^{\top}\left(\mathbf{v}_{b} \circ\left(\mathbf{s}_{b}-\hat{\mathbf{s}}_{b}\right)\left(\mathbf{s}_{b}-\hat{\mathbf{s}}_{b}\right)^{H} \circ \mathbf{v}_{b}^{H}\right) \mathbf{h}_{b}^{*} \\
& \stackrel{(a)}{=} \sum_{b=1}^{B} \frac{1}{N_{0, b}} \mathbf{h}_{b}^{\top} \mathbf{U}_{b} \mathbf{D}_{b} \mathbf{U}_{b}^{H} \mathbf{h}_{b}^{*} \\
& \stackrel{(b)}{=} \sum_{b=1}^{B} \frac{1}{N_{0, b}}\left|w_{b}\right|^{2}\left\|\mathbf{v}_{b} \circ\left(\mathbf{s}_{b}-\hat{\mathbf{s}}_{b}\right)\right\|^{2}
\end{aligned}
$$

where $(\cdot)^{*}$ is the conjugate operation, $\mathbf{U}_{b}$ is a unitary matrix, and $\mathbf{D}_{b}$ is the diagonal matrix with eigenvalues being the diagonal terms. In (14), (a) is due to the eigenvalue decomposition of the Hermitian matrix $\mathbf{v}_{b} \circ\left(\mathbf{s}_{b}-\hat{\mathbf{s}}_{b}\right)\left(\mathbf{s}_{b}-\hat{\mathbf{s}}_{b}\right)^{H} \circ \mathbf{v}_{b}^{H}$ [17], and (b) follows from the fact that due to the rank-1 property of $\mathbf{v}_{b} \circ\left(\mathbf{s}_{b}-\hat{\mathbf{s}}_{b}\right)\left(\mathbf{s}_{b}-\hat{\mathbf{s}}_{b}\right)^{H} \circ \mathbf{v}_{b}^{H}, \mathbf{D}_{b}$ has only one nonzero entry with value $\left\|\mathbf{v} \circ\left(\mathbf{s}_{b}-\hat{\mathbf{s}}_{b}\right)\right\|^{2}$. $w_{b}$ is defined as

$$
w_{b}=\mathbf{h}_{b}^{\top} \mathbf{u}_{b},
$$

where $\left\|\mathbf{u}_{b}\right\|^{2}=1$ is the eigenvector associated with that nonzero eigenvalue in D. By using (14), $M_{\Lambda}(t \mid \mathbf{H})$ becomes

$$
M_{\Lambda}(t \mid \mathbf{H})=\prod_{b=1}^{B} e^{\left(t+t^{2}\right) \frac{1}{N_{0}, b}\left|w_{b}\right|^{2}\left\|\mathbf{v}_{b} \circ\left(\mathbf{s}_{b}-\hat{\mathbf{s}}_{b}\right)\right\|^{2}} .
$$

We then approximate the PEP using Chernoff bound with $t=$ $-1 / 2$ [18], which yields

$$
f(\mathbf{s} \rightarrow \hat{\mathbf{s}}) \simeq f_{\mathrm{CB}}(\mathbf{s} \rightarrow \hat{\mathbf{s}})=\prod_{b=1}^{B} e^{-\gamma_{b} \frac{\left\|\mathbf{v}_{b} \circ\left(\mathbf{s}_{b}-\hat{\mathbf{s}}_{b}\right)\right\|^{2}}{4 \sigma_{x}^{2}}} .
$$

In (17), the effective SNR $\gamma_{b}$ is defined as

$$
\gamma_{b} \triangleq \frac{\left|w_{b}\right|^{2} \sigma_{x}^{2}}{N_{0, b}}
$$

where $\sigma_{x}^{2}$ denotes the variance of $\mathbf{x}$. Now, we define $\Delta_{b}$ as

$$
\Delta_{b}= \begin{cases}1, & \text { if otherwise, } \\ 0, & \text { if } \mathbf{v}_{b} \circ\left(\mathbf{s}_{b}-\hat{\mathbf{s}}_{b}\right)=\mathbf{0} .\end{cases}
$$

Then, according to (14), (17), and assuming $\gamma_{b}$ is the same for all the time instants such that the subscript $b$ can be omitted for simplicity, the diversity $d$ can be represented as follows:

$$
d=-\lim _{\gamma \rightarrow \infty} \frac{\log [f(\mathbf{s} \rightarrow \hat{\mathbf{s}})]}{\log [\gamma]}=\sum_{b=1}^{B} \Delta_{b}
$$

From (20), we see that once $T$ approaches infinity, we have $\mathbf{v}_{b}=\mathbf{1}$, and thus the PTC system achieves full diversity if $\left(\mathbf{s}_{b}-\hat{\mathbf{s}}_{b}\right) \neq \mathbf{0}$, for $b=1, \ldots, B$. However, since infinitely large $T$ is practically impossible, we need to investigate the appropriate value of $T$ to preserve the diversity.

For a good design of the PTC, the data packet is dispersed into all time instants and relay paths so that we have the 
column vector $\left(\mathbf{s}_{b}-\hat{\mathbf{s}}_{b}\right)$ with all entries nonzero. In this case, $\Delta_{b}=0$ happens when $\mathbf{v}_{b}=0$. The probability of having $\mathbf{v}_{b}=0$ is given by,

$$
P\left(\mathbf{v}_{b}=\mathbf{0}\right)=P\left(v_{b, r}=0 ; r=1, \ldots, R\right),
$$

which is also the probability of the $b$ th coded packet being erased. This probability is calculated in the next subsection.

\section{B. Erasure Statistics}

We first construct the relationship between the availability of an opportunistic link and a relay path. Then, the erasure probability $P\left(v_{b, r}=0\right)$ as a function of the waiting period $T$, number of opportunistic links $N_{r}$, and link availability $p$ is derived. As mentioned previously, each node simply performs sensing at the beginning of every sensing interval. Once the end-to-end delay exceeds the waiting period, the coded packet is discarded. Sequential sensing of the $N_{r}$ opportunistic links can be modeled as a Bernoulli trial, characterized by the geometrically distributed random, variable $G(1-p)$. Herein, we assume that the statistics of each opportunistic links is the same. Since the end-to-end delay is the summation of $N_{r}$ geometric random variables, it follows a negative binomial distribution $\mathrm{NB}(N, p)$ with probability mass function (PMF)

$$
P_{\mathrm{NB}}\left(x ; N_{r}, 1-p\right)=\left(\begin{array}{c}
x+N_{r}-1 \\
N_{r}-1
\end{array}\right) p^{N_{r}}(1-p)^{x} .
$$

When the end-to-end delay $\tau$ is shorter than $T$, the relay path is considered as available with probability given by [15]

$$
\begin{aligned}
P\left(v_{b, r}=1\right) & =P(\tau \leq T)=\sum_{\tau=N_{r}}^{T} P_{\mathrm{NB}}\left(\tau-N_{r} ; N_{r}, 1-p\right) \\
& =\sum_{\tau=N_{r}}^{T}\left(\begin{array}{c}
\tau-1 \\
N_{r}-1
\end{array}\right) p^{N_{r}}(1-p)^{\tau-N_{r}} .
\end{aligned}
$$

\section{Design Guideline of Waiting Period}

Although the exact erasure statistics can be derived by using the negative binomial distributions, as can be seen from (23), that expression fails to show clear relationship among the system parameters, e.g., the waiting periods $T$, the link availability $p$, and the resulting erasure statistics $P\left(v_{r, b}=0\right)$. Therefore, an simpler approximation is resorted. We define the Bernoulli random variable $u_{b, r, t} \in\{0,1\}$ as the indicator which represents the sensing result of $t$ th attempt of the $b$ th coded packet in the $r$ th relay path. $u_{b, r, t}=1$ and $u_{b, r, t}=0$ respectively imply that the $r$ th coded packet is forwarded or buffered. Note that if the coded packet arrives at the destination node at the $t^{\prime}$ th attempt, we have $u_{b, r, t}=0$ for $t^{\prime}<t \leq T$. The erasure probability can thus be given by

$$
\begin{aligned}
P\left(v_{b, r}=0\right) & =P\left(\sum_{t=1}^{T} u_{b, r, t} \leq N_{r}-1\right) \\
& =P\left(\sum_{t=1}^{T} u_{b, r, t} \leq\left(1-\frac{p T-N_{r}+1}{p T}\right) p T\right) \\
& \stackrel{(a)}{\leq} e^{\left(-\frac{1}{2} \frac{\left(p T-N_{r}+1\right)^{2}}{p T}\right)},
\end{aligned}
$$

where $(a)$ is due to the Chernoff bound. In particular, for random variable $\sum_{t=1}^{T} u_{b, r, t}$, the Chernoff bound states that

$$
P\left[\sum_{t=1}^{T} u_{b, r, t} \leq(1-\alpha) \mu\right] \leq e^{\left(-\frac{\alpha^{2} \mu}{2}\right)},
$$

where $\mu=\mathbb{E}\left[\sum_{t=1}^{T} u_{b, r, t}\right]=p T$ due to the independent and identical distribution of $u_{b, r, t}$, and $|\alpha| \leq 1$. By letting $\alpha=\frac{p T-N_{r}+1}{p T}$, we have (24).

With the aid of (24), the upper bound of the probability of the diversity loss defined in (21) can be given by

$$
\begin{aligned}
P\left(\mathbf{v}_{b}=0\right) & =P\left(v_{b, 1}=0\right) \cdots P\left(v_{b, R}=0\right) \\
& \leq \prod_{r=1}^{R} \exp \left(-\frac{1}{2} \frac{\left(p T-N_{r}+1\right)^{2}}{p T}\right) \\
& \leq \exp \left(-\frac{1}{2} \frac{\left(p T-N_{r^{\star}}+1\right)^{2}}{p T}\right),
\end{aligned}
$$

where the $r^{\star}$ th path is the path with largest number of relay nodes:

$$
r^{\star}=\max _{r=1, \ldots, R} N_{r}
$$

This upper bound of the probability of diversity loss is smaller than unity only if the exponent in (26) is negative. Since the necessary condition of the negative exponent is

$$
T>\frac{1}{p}\left(N_{r^{\star}}-1\right)
$$

which implies that the probability of diversity loss is bounded by a value smaller than unity, we thus propose the design guideline as

$$
T^{\star}=\left\lceil\frac{1}{p}\left(N_{r^{\star}}-1\right)\right\rceil+1
$$

where the second term in the right hand side, i.e., ' +1 ', is added to guarantee a positive waiting period even for the case of $N_{r}^{\star}=1$. As will be demonstrated in the next section, the design guideline (29) can simultaneously preserve the diversity of the end-to-end PTC transmissions and minimizes the transmission latency.

\section{Simulation Results}

In this section, the design guideline (29) is demonstrated by means of Monte Carlo simulations. We use QPSK modulation and DFT-based PTC. For simplicity, the destination node has perfect knowledge of the erasures $v_{b, r}$ and the path fading gains $h_{b, r}$. In practice, the erasures are jointly identified with the data decoding by using the joint sphere decoding algorithm [5] which only results in few $\mathrm{dB}$ SNR degradation. Both the number of relay paths and time instants are set to be three, i.e., $R=B=3$. We set the number of opportunistic links in the third path $N_{3}=4$ and $\left(N_{1}, N_{2}\right)$ are uniformly distributed with support $[1,4]$. We simulate the link availability $p=0.85$ based on the $15 \%$ spectrum utilization reported by FCC [19]. The double and triple spectrum utilizations, i.e., $p=0.7$ and $p=0.55$, are also simulated to model the scenarios where the CR technology greatly enhances the spectrum utilizations. 


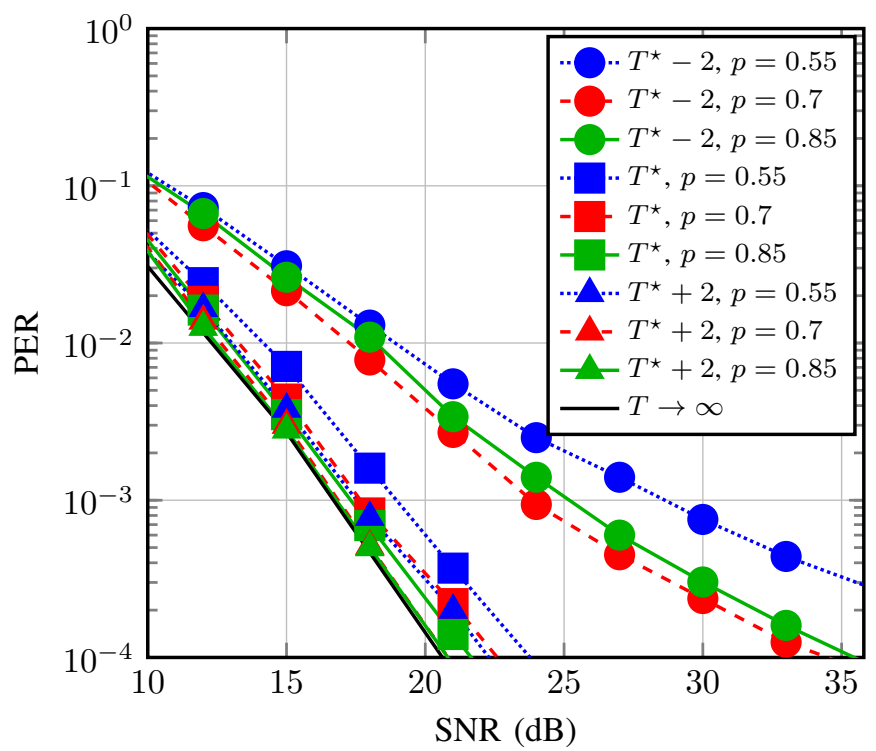

Fig. 3. Error rate performance of the end-to-end PTC transmission with various waiting periods. $R=3$ and $B=3$ are used. $N_{3}$ is assumed to be 4 and $\left(N_{1}, N_{2}\right)$ are uniformly-distributed with support $[1,4]$. Thus, for $p=$ $0.85,0.7$, and 0.55 , we respectively have $T^{\star}=5,6$, and 7 .

In this case, the design guideline in (29) provides $T^{\star}=5,6$, and 7 , respectively for $p=0.85,0.7$ and 0.55 .

Fig. 3 demonstrates the effectiveness of the design guideline (29) by showing the packet error rate (PER) performance. The solid, dashed, and dotted lines with markers are respectively used for $p=0.85, p=0.7$ and $p=0.55$. The solid line without marker indicates the case of infinity waiting period, i.e., $T \rightarrow \infty$, which serves as the lower bounds of all the settings. We can see that by using the proposed waiting period $T^{\star}$ defined in (29), the slopes of the curves are similar to the case of $T \rightarrow \infty$. We add or subtract the waiting periods by a value of 2 to show the change of the performance with longer or shorter waiting period. Although the error rate performance is slightly improved when we increase the waiting period, the diversity remains the same. Meanwhile, if the waiting period is less than $T^{\star}$, the diversity loss occurs, and the error rate increases drastically.

The trade-off between the transmission reliability and the latency is illustrated in Fig. (4). Without diversity loss, the diversity of the end-to-end PTC transmission with three relay paths is slightly less than 3 , due to the cascaded fading. We can see that the diversity gain from $T^{\star}$ to $T^{\star}+1$ is smaller than the diversity gain from $T^{\star}-1$ to $T^{\star}$, which shows the effectiveness of the proposed waiting period. Although the diversity loss still remains when $T^{\star}$ is selected, such loss is small compared with the case of no diversity loss. Thus, the design guideline $T^{\star}$, derived in (29), strikes a good balance between the transmission latency and reliability under the condition of negligible diversity loss.

\section{CONClusion}

In this paper, we investigate the reliability and latency trade-off of the end-to-end PTC transmission in the CRAHNs. The PEP is derived to obtain diversity loss as a function of

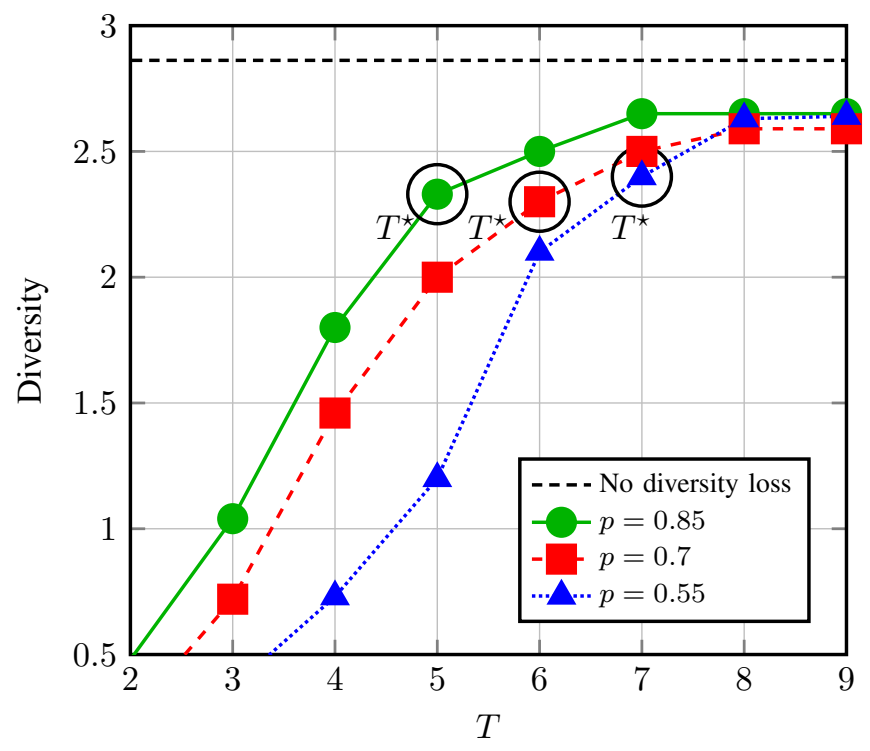

Fig. 4. Diversity vs. waiting period of the end-to-end transmissions with various link availabilities $p . R=3$ and $B=3$ are used. $N_{3}$ is assumed to be 4 and $\left(N_{1}, N_{2}\right)$ are uniformly-distributed with support $[1,4]$. Thus, for $p=0.85,0.7$, and 0.55 , we respectively have $T^{\star}=5,6$, and 7 .

erasure statistics. The erasure statistics is approximated by the Chernoff bound, which yields a simple and clear expression to allow us to propose a design criterion of the waiting period, i.e., the limit of the transmission latency. Numerical demonstrations show that the propose design criterion (29) strikes a good balance between the transmission latency and reliability.

\section{ACKNOWLEDGEMENT}

The support from Ministry of Science and Technology (MOST), Taiwan is greatly appreciated.

\section{REFERENCES}

[1] J. Mitola III, "Cognitive radio for flexible mobile multimedia communications," in Proc. 6th IEEE Int. Workshop on Mobile Multimedia Communications (MOMUS99), Nov. 1999.

[2] K.-C. Chen and R. Prasad, Cognitive Radio Networks. Chichester, UK: John Wiley \& Sons, 2009.

[3] K.-C. Chen, B. K. Cetin, Y.-C. Peng, N. Prasad, J. Wang, and S. Lee, "Routing for cognitive radio networks consisting of opportunistic links," in Wirel. Commun. Mob. Comput. (John Wiley), Aug. 2009, pp. $451-466$.

[4] I.-W. Lai, C.-H. Lee and K.-C. Chen, "A virtual MIMO path-time code for cognitive ad hoc networks," IEEE Commun. Lett., vol. 17, no. 1, pp. 4-7, Jan. 2013.

[5] I.-W. Lai, C.-L. Chen, C.-H. Lee, K.-C. Chen and E. Biglieri, "Endto-end virtual MIMO transmission in ad hoc cognitive radio networks," IEEE Trans. Wireless Commun., vol. 13, no. 1, pp. 330-341, Jan. 2014.

[6] B. Mumey, J. Tang, I. R. Judson, and D. Stevens, "On routing and channel selection in cognitive radio mesh networks," IEEE Trans. Veh. Technol., vol. 61, no. 9, pp. 4118-4128, Nov. 2012.

[7] M. Caleffi, I. F. Akyildiz, and L. Paura, "OPERA: Optimal routing metric for cognitive radio ad hoc networks," IEEE Trans. Wireless Commun., vol. 11, no. 8, pp. 2884-2894, Apr. 2012.

[8] K.-C. Chen and S. Lien, "Machine-to-machine communications: Technologies and challenges," Ad Hoc Networks, [Online]. Available: http://www.sciencedirect.com/science/article/pii/S1570870513000395

[9] S.-C. Lin and K.-C. Chen, "Spectrum aware opportunistic routing in cognitive radio networks," in Proc. IEEE Glob. Comm. Conf. (GLOBECOM), Miami, FL, USA, Dec. 2010, pp. 1-6. 
[10] K. R. Chowdhury and I. F. Akyildiz, "CRP: A routing protocol for cognitive radio ad hoc networks," IEEE J. Sel. Areas Commun., vol. 29 , no. 4, pp. 794-804, Apr. 2011.

[11] E. Biglieri, Coding for Wireless Channels. Springer, 2005.

[12] M. K. Marina and S. R. Das, "On-demand multipath distance vector routing in ad hoc networks," in Proc. 2001 IEEE Int. Conf. on Network Protocol (ICNP), Nov. 2001, pp. 14-23.

[13] P. Djukic and S. Valaee, "Reliable packet transmissions in multipath routed wireless networks," in IEEE trans. Mobile Comput., vol. 4, no. 5, pp. 548-559, May, 2006.

[14] W.-C. Ao and K.-C. Chen, "End-to-end HARQ in cognitive radio network," in Proc. IEEE Wireless Commun. and Networking Conf. (WCNC), Jul. 2010, pp. 1-6.

[15] P.-Y. Chen, W.-C. Ao, and K.-C. Chen, "Rate-delay enhanced multipath transmission scheme via network coding in multihop networks," IEEE Commun. Lett., vol. 16, no. 3, pp. 281-283, Mar. 2012.

[16] I.-W. Lai, C.-H. Lee, K.-C. Chen, and E. Biglieri, "Performance of pathtime codes for end-to-end transmission in ad hoc multihop networks," in Proc. IEEE Int. Symp. Information Theory (ISIT), Honolulu, HI, USA, June 2014, pp. 66-70.

[17] V. Tarokh, N. Seshadri, and A. R. Calderbank, "Space-time codes for high data rate wireless communication: Performance criterion and code construction," IEEE Trans. Inf. Theory, vol. 44, no. 2, pp. 744-765, Mar. 1998.

[18] G. Caire, G. Taricco, and Ezio Biglieri, "Bit-interleaved coded modulation," IEEE Trans. Inf. Theory, vol. 44, no. 3, pp. 927-946, May 1998.

[19] First Report and Order, Federal Communication Commission Std, FCC 02-48, Feb. 2002 